\title{
Circuit/system design space characterization of EER-based transmitter for 802.11a WLAN Standard
}

\author{
J. Marchán ${ }^{1}$, E. Barba ${ }^{1}$, L. Marco ${ }^{1}$, D. Maksimović ${ }^{2}$ and E. Alarcón ${ }^{1}$ \\ ${ }^{1}$ Energy Processing Integrated Circuits (EPIC) group \\ ${ }^{2}$ Colorado Power Electronics Center - CoPEC \\ Department of Electronic Engineering \\ UPC BarcelonaTech, 08034 Barcelona, Spain \\ e-mail: ealarcon@eel.upc.edu \\ Electrical and Computer Engineering Department \\ University of Colorado, Boulder, CO, 80309 USA \\ e-mail:maksimov@colorado.edu
}

\begin{abstract}
Due to the increase of required bandwidth, the newer generation of communication systems uses spectrum-efficient digital modulations that involve non-constant envelope RF signals. A technique that potentially can concurrently provide high efficiency and linearity is the Envelope Elimination and Restoration (EER) transmitter architecture which is based on the decomposition of the transmitted signal in a broadband baseband envelope (processed by an envelope-tracking switching power converter) and a narrowband phase-modulated signal (processed by a class-E switch-mode RFPA) and the subsequent restoration (through wideband adaptive RF PA supply). In this work, a behavioral system-level characterization of how circuitlevel effects (namely limited bandwidth and switching effects) affect the overall communication system performance metrics (characterized by spectra, eye diagrams, signal constellations, EVM and BER) of a 802.11a WLAN system is presented.
\end{abstract}

\section{INTRODUCTION}

RF power amplifiers (RF PAs) are the most dominant power-consuming devices in battery-operated terminals for communication systems [1]. Systems like GSM or Bluetooth employ constant envelope RF signals which allow highefficiency RF amplification by means of class-E switch-mode switching amplifiers, since there is no need of linearity. Due to the increasing bandwidth demands associated to the evolution of communication systems to manage multimedia information for which very high data rates are needed to transfer images, videos and audio, the newer generation of communication systems (e.g. EDGE, W-CDMA, WLAN or $4 \mathrm{G}$ standards) use spectrum-efficient digital modulations that involve nonconstant envelope RF signals. In principle, to amplify nonconstant envelope RF signals a linear power amplifier is required, which inherently exhibits low DC-to-RF efficiency.

Different transmitter architectures have been proposed to improve the efficiency while guaranteeing linearity. One of the most promising techniques is the Envelope Elimination and Restoration (EER) technique [2]. The EER technique is based on the decomposition of the transmitted signal in the two components of the polar representation of complex signals: the broadband baseband envelope signal and the narrowband phase-modulated signal. A high efficiency switch-mode RF PA is driven by the RF phase signal, whereas the envelope is amplified by an efficient baseband power amplifier, typically a buck-derived switching power converter. Finally, the recombination of the envelope and phase signals is accomplished by drain modulation of the RF PA, obtaining ideally an amplified replica of the input signal. The circuitlevel scheme of the EER architecture is shown in Fig. 1.

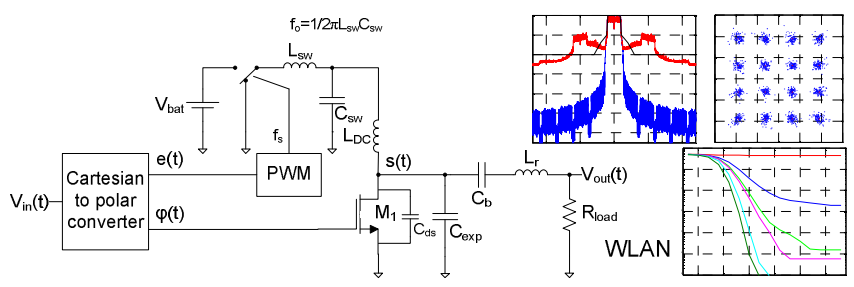

Figure 1. Circuit-level representation of EER transmitter

Several design challenges still preclude the complete success of this technique. Time misalignments in the two signal paths, AM-PM distortion at the RF PA, and limited performance of the envelope path all degrade the system linearity. One of the key remaining challenges for a successful realization of such system is the practical implementation of efficient, wide-bandwidth baseband envelope-tracking power converter [3]. Hitherto, the study of a Class-E PA as a RF amplifier [4], considering separately limited envelope's path bandwidth and time mismatch between paths [5,6,7] and adding AM/AM curve to model restoration [8] with a WLANlike signal have been performed. With a two-tone test, an EER architecture with a switching power converter as envelope amplifier has also been evaluated [9]. Nevertheless, a design space characterization of the switching and filtering effects of a switching power converter as envelope amplifier is of key importance, and is particularly critical in face of the stringent specifications of the WLAN standard.

In this work, a study of how circuit-level effects such as the switching frequency of the envelope-tracking power converter together with its limited bandwidth as well as the delay 
misalignment between the two polar paths affect the overall system-level communications scheme quality of a WLAN system (upon its constellation, BER, EVM, spectra) is presented. Section II reviews the EER architecture and identifies the open design parameters for the switching power converter. Section III reviews the 802.11a standard and shows nominal operation samples of WLAN signals and characteristics. Section IV finally characterizes the design space associated to the switching converter inherent performance limitations (bandwidth limits and switching), through a complete design space exploration, upon systemlevel performance metrics, e.g. spectra, constellation, eye diagram, EVM, BER.

\section{EER ARCHITECTURE}

The main principle of an EER transmitter technique is to split the input signal, of non-constant envelope, into its two polar components: envelope and phase, thereby obtaining a baseband signal the amplitude of which corresponds to the input signal envelope and a constant envelope signal with the phase modulation of the input signal. Thus, they can be amplified in an efficient manner separately by means of switching amplifiers.

In this work, the polar transmitter is implemented by means of an ideal Class-E switching amplifier, a delay compensator, and a PWM-driven buck-based switching power converter. The Class-E RFPA non idealities are not considered, an ideal linear supply to RF output modulation is assumed as well as an ideal Cartesian to polar conversion. The delay compensator inserts a pure transport delay on the phase path. In Fig. 2, the behavioural level Simulink model used is shown.

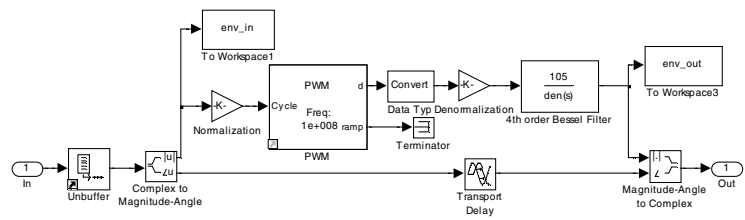

Figure 2. EER model with a switching power converter and delay phase compensator

The two open design parameters that characterize the switching power converter design space are its switching frequency $\left(f_{s}\right)$ and the cut-off frequency $\left(f_{o}\right)$ of the LC lowpass filter.

\section{WLAN 802.11A STANDARD}

The $802.11 \mathrm{a}$ amendment to the original standard was ratified in 1999. It operates at $5 \mathrm{GHz}$ band, and defines 64 OFDM subchannels, although only 48 of them are used for data and another 4 for pilot signals, because the remaining 12 are used for synchronization and guard effects. It provides a wireless LAN with data payload communication capabilities of $6,9,12,18,24,36,48$, and $54 \mathrm{Mbit} / \mathrm{s}$ rates, automatically selected depending on the channel quality, reserving higher data rates for good quality channels. Each of the 48 OFDM subcarriers is modulated using BPSK, QPSK, 16-QAM or 64QAM. Forward Error Correction (FEC) coding (convolutional coding) is used with a coding rate of $1 / 2,2 / 3$, or $3 / 4$. The modulation method and the coding rate depend upon the desired rate and the available Signal-to-Noise Ratio (SNR).

The main advantage of this modulation is its high spectrum efficiency since the orthogonality between subcarriers allows them to overlap. On the other hand, the main drawback is the high peak-to-average ratio (PAR) caused by the large number of added independent subcarriers with random phase.

Eight different operating modes have been defined, from the lowest data-rate mode (mode 1: 6Mbit/s, BPSK modulated with a coding rate of $1 / 2$ ) to the highest data-rate mode (mode 8: 54Mbit/s, 64-QAM modulated with a coding rate of 3/4).

Fig. 3 and 4 show respectively the time-domain envelope signal and its associated output signal spectrum of a WLAN system in order to have a reference to compare to the signals later obtained when incorporating the EER polar transmitter.

\section{SWITCHING AND FILTERING EFFECTS UPON WLAN SIGNAL}

A circuit/system design space exploration of an EERbased transmitter has been carried out for the WLAN standard. In the following the main results operating at mode 5 (24 $\mathrm{Mbit} / \mathrm{s}$ ) are presented. More results for the remaining modes can be found in [10].

For illustration purposes, Fig. 5 shows the envelope signal before the restoration. The configuration used is one of the bests in terms of signal fidelity since the switching frequency is in the high range $(100 \mathrm{MHz})$ and the cut-off frequency $(30 \mathrm{MHz})$ is almost logarithmically spaced from signal bandwidth $(10 \mathrm{MHz})$ and switching frequency in order to minimize distortion while rejecting the switching ripple.

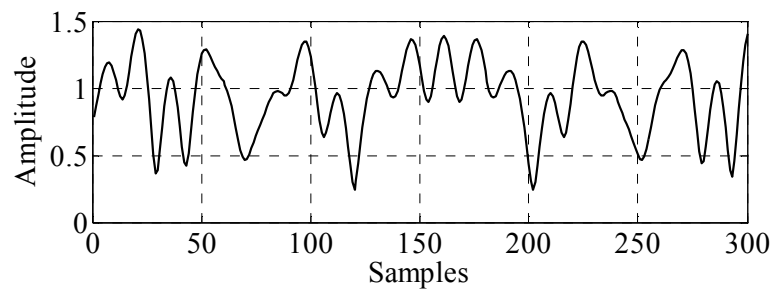

Figure 3. WLAN envelope time domain sample

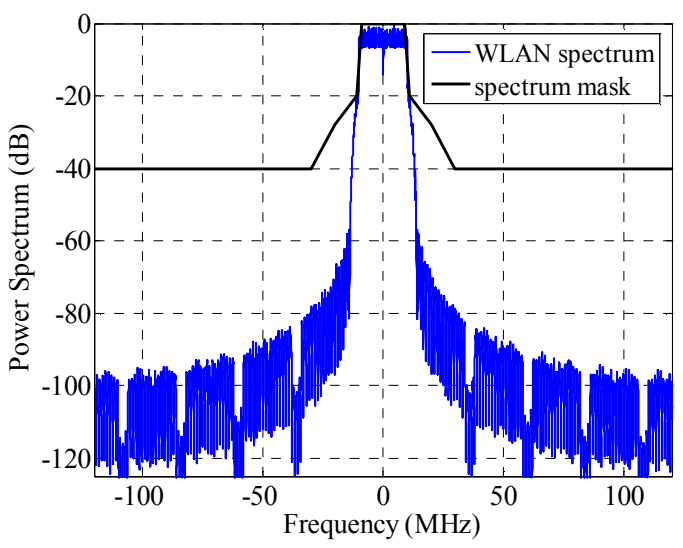

Figure 4. Power spectrum of a ideal radiated WLAN signal 


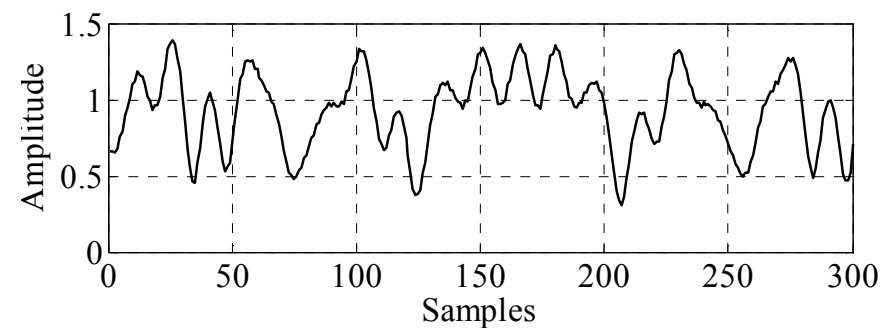

Figure 5. WLAN output envelope for $\mathrm{f}_{0}=30 \mathrm{MHz}$ and $\mathrm{f}_{\mathrm{s}}=100 \mathrm{MHz}$

In Fig. 6, it can be observed that few of the configurations fulfill the spectrum mask (a performance specification which is common to all 8 WLAN modes). Note that, despite only a $4 \times 4$ coarse sweep is shown, the actual exploration considers a much finer tessellation. The configurations of the last column fail the mask due to the spectral regrowth produced by the limited envelope path bandwidth (cut-off frequency) and delay mismatches. The alias in the switching frequency is not rejected at all in the configurations in the diagonal (same cutoff and switching frequencies). The design space subset of configurations that fulfill the mask represents the possible candidate solutions in which receiver-level performance metrics (constellation, and quantitatively EVM) will be evaluated.

In Fig. 7 it can be observed that the best configurations at the receiver end in terms of constellation diagram (which requires implementing a complete receiver) are the ones with high switching and cut-off frequency. The reason for this behaviour is the inclusion of the reception filter which is mandatory to avoid aliasing, but also rejects the switching alias.

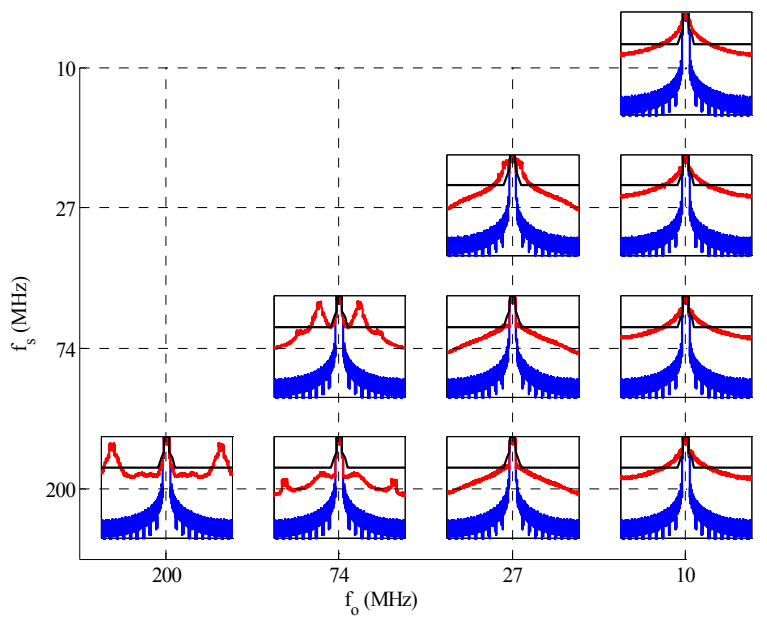

Figure 6. WLAN TX signal spectra for the complete $(f s, f o)$ design space

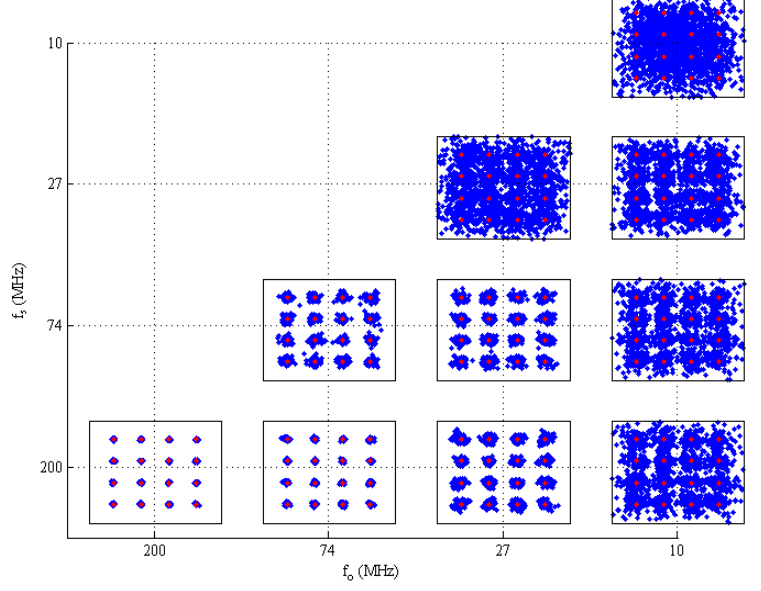

Figure 7. 16-QAM scatter plots for the complete $(f s, f o)$ design space

Fig. 8 quantifies the dispersion qualitatively observed in Fig. 7 (signal constellations) by means of the $\mathrm{EVM}_{\mathrm{rms}}$ calculation. Fig 8 (a) considers an LC Bessel filter of $4^{\text {th }}$ order. Note that, for this simple case, only a very reduced subset of design parameters fulfill the standard EVM requirements. By modifying the envelope tracking converter to incorporate a Butterworth $6^{\text {th }}$ filter, the EVM metric result as in Fig 8 (b), thereby opening a broader window of candidate solutions. A complete final design exploration should evaluate, for this input design subspace, converter power losses and system efficiency so as to identify the optimum design, which is the scope of future work.

Eventually, the quality of the signal at the receiver is studied through the Bit Error Rate (BER). It can be observed in figure 10 that the BER of some configurations converge to a constant saturation value for high SNRs. This constant value means that the distortion introduced by the transmitter generates incorrigible errors, thereby decreasing the performance quality.

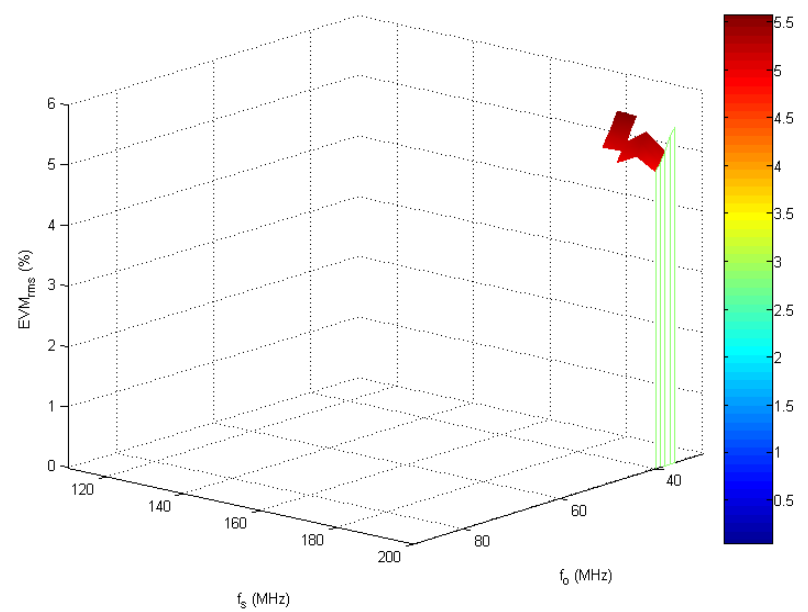

(a) 


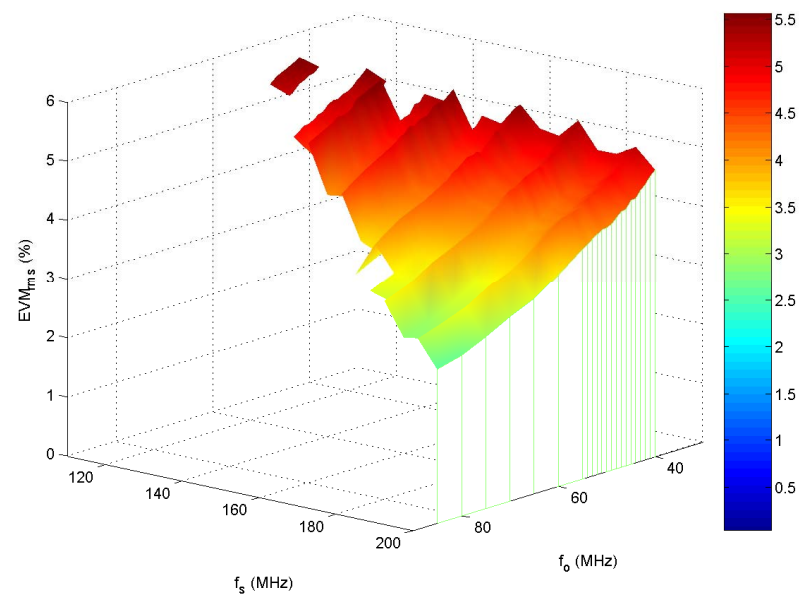

(b)

Figure 8. 16-QAM EVMrms for configurations that fulfil mode 5 EVM standard requirements. (a) Bessel $4^{\text {th }}$ order filter and (b) Butterworth $6^{\text {th }}$ order filter in the switching converter envelope tracker

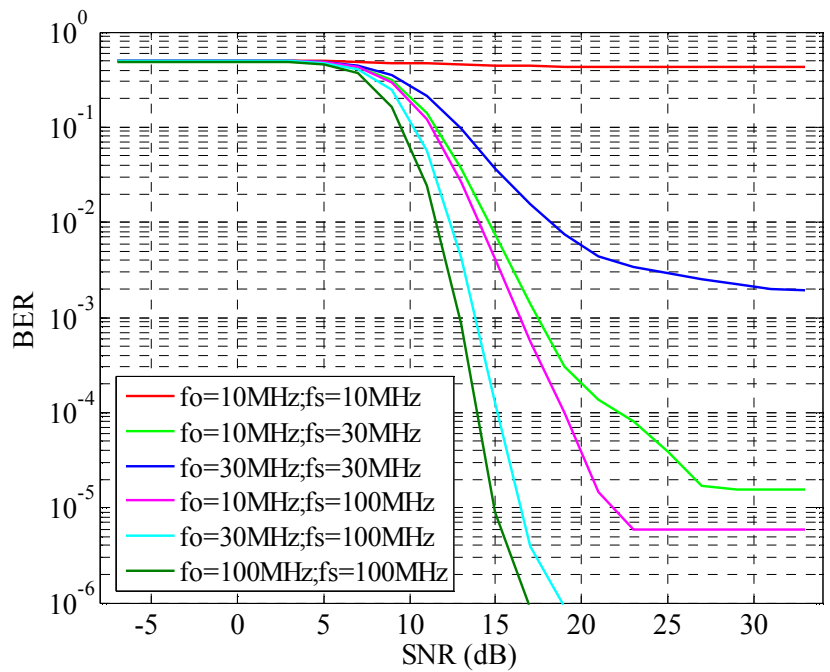

Figure 9. mode 5 BER vs. SNR plot for different EER TX configurations

\section{CONCLUSIONS}

In this work a polar EER RF transmitter architecture has been modeled with a switching amplifier circuit-level model for the envelope amplifier. It has been integrated in a nonconstant envelope communication system (802.11a WLAN standard) model implemented at behavioral level via Simulink. The model has been tested for several different simulation conditions, thereby characterizing the complete system through a design space exploration that bridges circuitlevel open design variables $\left(f_{s}, f_{o}\right)$ and communications system-level performance metrics (spectra, signal constellations, EVM and BER).

For the reduced subset of converter design parameters which fulfill the spectrum mask, receiver-level performance in terms of EVM has been evaluated for two switching converter alternatives, namely using a Bessel $4^{\text {th }}$ order or Butterworth $6^{\text {th }}$ order filter.
Taking into account that higher switching frequency would lead to unacceptable switching losses, compromising overall efficiency, future research will use the same circuit/system codesign framework to evaluate higher performance circuits, for instance evaluating other topologies as envelope amplifiers such as multilevel buck converters, linear assisted switching power amplifiers, adding feedback or predistortion, and employing asynchronous sigma-delta modulation instead of PWM. The final aim is to assess their combined performance in terms of receiver-level performance metrics, which will constitute a design-space constraint to the merit figure characterizing the efficiency of the overall system.

Acknowledgements. Partial support by project TEC2007-67988-C0201 and by project RUE CSD2009-00046, Consolider-Ingenio 2010 Programme from the Spanish Ministry of Science and Innovation and EU FEDER funds.

\section{REFERENCES}

[1] F. Raab, P. Asbeck, S. Cripps, P. Kenington, Z. Popovic, N. Pothecary, J. Sevic and N. Sokal, "Power amplifiers and transmitters for RF and microwave," Microwave Theory and Techniques, IEEE Transactions on, vol. 50, pp. 814-826, Mar 2002.

[2] F. Raab, "Intermodulation distortion in Kahn-technique transmitters," Microwave Theory and Techniques, IEEE Transactions on, vol. 44, pp. 2273-2278, Dec 1996.

[3] V. Yousefzadeh, E. Alarcon and D. Maksimovic, "Three-level buck converter for envelope tracking in RF power amplifiers," Applied Power Electronics Conference and Exposition, 2005. APEC 2005. Twentieth Annual IEEE, vol. 3, pp. 1588-1594 , 610 March 2005.

[4] A. Diet, C. Berland, M. Villegas and G. Baudoin, "EER architecture specifications for OFDM transmitter using a class E amplifier," Microwave and Wireless Components Letters, IEEE, vol. 14, pp. 389-391, Aug 2004.

[5] W. Liu, J. Lau and R. Cheng, "Considerations on applying OFDM in a highly efficient power amplifier," Circuits and Systems II: Analog and Digital Signal Processing, IEEE Transactions on [see also Circuits and Systems II: Express Briefs, IEEE Transactions on], vol. 46, pp. 1329-1336, Nov 1999.

[6] D. Rudolph, "Out-of-band emissions of digital transmissions using Kahn EER technique," Microwave Theory and Techniques, IEEE Transactions on, vol. 50, pp. 1979-1983, Aug 2002.

[7] D. Rudolph, "Kahn EER technique with single-carrier digital modulations," Microwave Theory and Techniques, IEEE Transactions on, vol. 51, pp. 548-552, Feb 2003.

[8] G. Baudoin, C. Berland, M. Villegas and A. Diet, "Influence of time and processing mismatches between phase and envelope signals in linearization systems using envelope elimination and restoration, application to Hiperlan2," Microwave Symposium Digest, 2003 IEEE MTT-S International, vol. 3, pp. 2149-2152, June 2003.

[9] L. Marco, E. Alarcon and D. Maksimovic, "Effects of switching power converter nonidealities in envelope elimination and restoration technique," Circuits and Systems, 2006. ISCAS 2006. Proceedings. 2006 IEEE International Symposium on, pp. 4, 21-24 May 2006.

[10] J. Marchán, "Modelling of 802.11a Standard polar transmitter for characterization of circuit non idealities," Master's thesis, UPC, Jul. 2008. 